\title{
ASPECTOS FLORÍSTICOS E ECOLÓGICOS DA VEGETAÇÃO CAMPESTRE DO MORRO DA POLÍCIA, PORTO ALEGRE, RS, BRASIL ${ }^{1}$
}

\author{
Ilsi Iob Boldrini ${ }^{2}$ \\ Silvia T. S. Miotto ${ }_{3}^{3}$ \\ Hilda M. Longhi-Wagner ${ }_{3}$ \\ Valério De P. Pillar \\ Katia Marzall
}

Recebido em 27/9/96. Aceito em 24/3/98

\begin{abstract}
RESUMO -(Vegetação campestre do Morro da Polícia, Porto Alegre, RS, Brasil). Estudou-se a variação da composição florística da vegetação campestre e suas relações com altitude e exposição no Morro da Polícia, de origem granítica, em Porto Alegre, Rio Grande do Sul, a $30^{\circ} \mathrm{S} \mathrm{e} 51^{\circ} \mathrm{W}$. O levantamento foi realizado de novembro de 1992 a novembro de 1994. A presença e freqüência de espécies foi avaliada usando o método de pontos sobre transecções em 19 unidades de vegetação, as quais foram delimitadas com base na exposição da encosta (N, S, L, O) e altitude ( 160 a 280m). Os dados foram submetidos a análise multivariada de agrupamentos e ordenação. Nas transecções foram observadas 189 espécies, sendo Poaceae, Asteraceae e Apiaceae as famílias mais freqüentes e Trachypogon montufari e Schizachyrium tenerum as espécies dominantes. A análise de agrupamentos e de ordenação revelou conexões entre variação da vegetação e fatores altitude e exposição solar. Aristida laevis e Sorghastrum albescens são características de comunidades mais expostas à radiação solar, enquanto Agenium villosum e Eryngium eriophorum o são de comunidades menos expostas. Vernonia nudiflora e Axonopus suffultus caracterizam as áreas mais elevadas do morro, enquanto Eupatorium ligulifolium e Sorghastrum albescens as mais baixas.
\end{abstract}

Palavras-chave: altitude, análise multivariada, exposição solar, fitossociologia, vegetação campestre
ABSTRACT - (Floristics and ecological aspects of a grassland vegetation on Morro da Polícia, Porto Alegre, RS, Brazil). We studied floristic composition variation and its conections to elevation and exposure in the grassland vegetation of Morro da Polícia, a hill of granitic origin in the city of Porto Alegre, Rio Grande do Sul, $30^{\circ} \mathrm{S} \mathrm{e} 51^{\circ} \mathrm{W}$. The survey took place from November 1992 to November 1994. Species presence and frequency were assessed by using the point method on transects in 19 vegetation units, which were delimited by slope exposure and elevation ( 160 to $280 \mathrm{~m}$ ). Data were subjected to cluster analysis and ordination. Based on data from transects we detected 189 species, mainly from the Poaceae, Asteraceae and Apiaceae families, with Trachypogon montufari and Schizachyrium tenerum as dominant

\footnotetext{
Trabalho apresentado no XLVII Congresso Nacional de Botânica

Departamento de Botânica, Instituto de Biociências, UFRGS. Av. Paulo Gama, 40, CEP 90040-900, Porto Alegre, RS

Bolsista do CNPq

Estudante de Mestrado em Fitotecnia, Agronomia, UFRGS
} 
species. Cluster analysis and ordination revealed conections of vegetation variation with elevation and exposure. Aristida laevis and Sorghastrum albescens are characteristic of more exposed comunities (N and NE slopes), while Agenium villosum and Eryngium eriophorum are from less exposed communities (on S and SW slopes). Vernonia nudiflora and Axonopus suffultus are characteristic of higher elevation areas on the hill, while Eupatorium ligulifolium and Sorghastrum albescens are characteristic of lower elevation areas.

Key words: elevation, exposure, grassland, multivariate analysis, phytosociology

\section{Introdução}

A vegetação dos campos do Morro da Polícia, elevação esta que faz parte do complexo de morros graníticos que circundam Porto Alegre, se destaca pela riqueza em espécies de diferentes famílias e pela sua beleza, o que já foi salientado por Rambo (1956). Esse complexo corresponde à projeção mais setentrional do Escudo Riograndense que, geologicamente, faz parte da Serra do Mar brasileira (Rambo 1954). Segundo Teixeira et al. (1986), a vegetação do município de Porto Alegre está classificada como Área de Tensão Ecológica, caracterizando um ecótono entre Áreas de Formações Pioneiras e Região da Floresta Estacional Semidecidual.

Entre os 11 tipos de formações edáficas presentes nestes morros salientados por Rambo (1956), estão o campo limpo, campo sujo e vassourais, formações situadas no topo, nas quais foi realizado o presente trabalho. O restante da área é ocupado por diferentes tipos de matas e capoeiras.

A flora dos arredores de Porto Alegre foi estudada por Teodoro Luis (1960), onde o autor fornece chaves e ilustrações das espécies, porém não especifica detalhadamente os locais de ocorrência. Mais recentemente destacam-se os trabalhos de Aguiar et al. (1986) e Mohr (1995). O primeiro deles é um levantamento florístico de todos os morros graníticos da grande Porto Alegre, que fornece, além de listagem dos 865 táxons encontrados, análise visual dos diferentes aspectos fitofisionômicos da área, com detalhamento maior da vegetação florestal. O segundo apresenta zoneamento de todas as comunidades vegetais presentes especificamente no Morro Santana, onde o estrato herbáceo foi amostrado com o método de pontos, resultando em listagem de 114 espécies, com predomínio das famílias Poaceae e Asteraceae. Nesse estrato foram identificadas duas comunidades, caracterizadas principalmente por gramíneas e, em menor escala, por ciperáceas e umbelíferas.

Este trabalho tem como objetivo caracterizar detalhadamente a vegetação campestre do Morro da Polícia, a partir de resultados de levantamento fitossociológico, estudando a sua composição florística e a influência sobre essa, de fatores físicos, como altitude e exposição solar.

\section{Material e métodos}

O Morro da Polícia está localizado na região fisiográfica da Depressão Central do Rio Grande do Sul (Borges Fortes 1959), no município de Porto Alegre, entre as coordenadas $30^{\circ} \mathrm{S}$ e $51^{\circ} \mathrm{W}$, sendo de origem granítica, com altitudes de até $280 \mathrm{~m}$. A área de vegetação campestre, onde foi realizado o trabalho, é de aproximadamente 110ha. 
Para a sistematização do trabalho de campo, as áreas de vegetação foram delimitadas através de imagem de satélite LANDSAT em transparência no equipamento PROCOM e a geração de um plano de informação para o SGI (Sistema Geográfico de Informação), incluindo curvas de nivel a cada $20 \mathrm{~m}$ de altitude. A plotagem foi realizada em carta de 1:50.000. Sobre esse mapa foram marcadas 18 transecções dispostas longitudinalmente a partir do ponto mais alto do morro até o final do dominio da formação campestre (Fig. 1). As transecções foram classificadas quanto à exposição em: norte (4 transecções), sul (3), leste (6) e oeste (5), as quais foram subdivididas em segmentos de comprimento variável, cada um deles delimitado por duas curvas de nivel consecutivas. Através da combinação de exposição (N: norte, S: sul, L: leste, O: oeste) e altitude $(1: 160-179 \mathrm{~m}, 2: 180-199 \mathrm{~m}, 3: 200-219 \mathrm{~m}, 4: 220-239 \mathrm{~m}, 5: 240-259 \mathrm{~m}$, 6:260-280m) foram definidas 19 unidades de vegetação (N2, N3, N4, N5, N6, S4, S5, S6, L1, L2, L3, L4, L5, L6, O2, O3, O4, O5, O6), aqui consideradas como comunidades vegetais.

Para a realização do levantamento de campo foi usado o método de pontos (Levy \& Madden 1933), amplamente discutido por Mantovani \& Martins (1990), em intervalos aproximados de $1 \mathrm{~m}$, totalizando 2.829 pontos. Foi verificada a presença/ ausência das espécies, calculada a freqüência absoluta das mesmas e com esses dados obtida a diversidade florística pelo índice de Shannon (Pielou 1975).

O levantamento foi iniciado em novembro de 1992 e finalizado em novembro de 1994. Os levantamentos foram realizados durante a primavera, verão e outono,

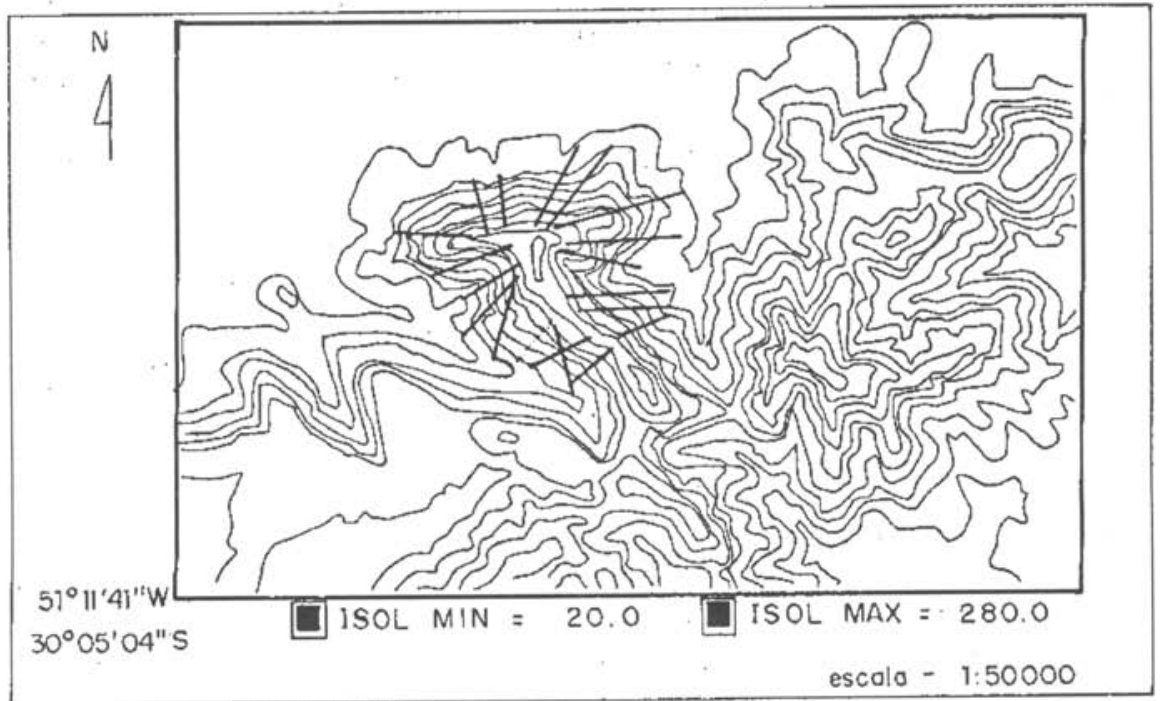

Figura 1. Localização do Morro da Policia, com as curvas de nivel e as transecções utilizadas no levantamento a campo. Porto Alegre, RS. 
procurando contemplar diferentes períodos de floração. Para complementação da listagem florística, foram realizadas observações e coletas fora das transecções.

Os dados das comunidades (uma matriz de 189 espécies por 19 comunidades vegetais) foram submetidos a análises multivariadas de agrupamentos, usando o critério de variância mínima, a partir de matriz de distâncias de corda e de ordenação pelo método de coordenadas principais, com distância de corda como medida de semelhança (Pielou 1984; Podani 1994). Foram utilizados diferentes métodos, com o uso do software SYNCSA (Pillar 1995), versão atualizada da originalmente descrita em Pillar \& Orlóci (1993). Como resultado das análises, foram elaborados um dendrograma e um diagrama de dispersão de comunidades.

\section{Resultados e discussão}

Composição florística - Com base nas transecções como um todo, foram identificadas 189 espécies, 99 gêneros e 26 famílias. As Poaceae participam com 72 espécies (38\%), as Asteraceae com 48 (25\%) e as Fabaceae com 25 (13\%). Seguem-se famílias com pequeno número de espécies, como as Rubiaceae e as Cyperaceae, com $7(3,7 \%)$, respectivamente, e as Apiaceae com 5 (2,6\%). É interessante observar que as três primeiras famílias totalizam $76 \%$ do total de espécies estudadas. Nas coletas feitas fora das transecções foram encontradas mais 105 espécies. A lista completa está na Tab. 1.

Com base na freqüência absoluta (FA) das espécies, verifica-se grande predomínio das gramíneas, o que confere com a situação fisionômica de campo limpo: Poaceae com 66,51\% das espécies; Asteraceae com 11,06\%; Apiaceae com 10,84\%; Fabaceae com 3,21\% Cyperaceae com 1,20\% e outras famílias com 7,18\%.

Ao comparar a participação das famílias quanto ao número de espécies com a freqüência absoluta das mesmas, verifica-se que Poaceae e Asteraceae mantém a mesma posição, enquanto que Fabaceae é substituída por Apiaceae. Isto, provavelmente, pode ser explicado pelo fato de que as espécies de Apiaceae formarem grandes populações, enquanto as espécies de Fabaceae encontram-se isoladas ou formando pequenas populações.

A área de campo é caracterizada por Trachypogon montufari e Schizachyrium tenerum (Poaceae) com 94,7\% de presença nas transecções, seguidas por Andropogon lateralis (Poaceae) e Eryngium horridum (Apiaceae) com 89,5\%, Elyonurus candidus, Sorghastrum albescens (Poaceae) e Eryngium pristis (Apiaceae) com 84,2\%, Agenium villosum com 78,9\%, Axonopus argentinus, Axonopus siccus, Schizachyrium imberbe, Paspalum plicatulum (Poaceae) e Baccharis sessiliflora (Asteraceae) com 68,4\%.

A diversidade florística, calculada pelo índice de Shannon $(\mathrm{H})$, tomando por base a frequiência das espécies calculada com todos os pontos sobre transecções na área estudada, foi de 4,01 e a riqueza ( $\mathrm{R}$ ou diversidade máxima $\mathrm{H}_{\max }$, Pielou 1975) com os mesmos dados foi de 5,24, resultando num índice de eqüidade (E) de 0,765. Se também forem consideradas as 105 espécies raras, encontradas somente fora das transecções, e a elas for atribuída a freqüência mínima de um ponto, a diversidade Shannon, a riqueza e a eqüidade seriam respectivamente 4,19, 5,68 e 0,737. 
Tabela 1. Familias e espécies encontradas dentro e fora das transecções, com a respectiva freqüência absoluta (FA), baseada em 2829 pontos. Morro da Policia, Porto Alegre, RS, BR.

\begin{tabular}{|c|c|c|c|}
\hline Spp. & FA & Spp. & FA \\
\hline ACANTHACEAE & & HYPERICACEAE & \\
\hline Justicia cf. flexuosa (Nees) Wass \& L.B. Sm. & & Hypericum connatum Lam. & \\
\hline AMARANTHACEAE & & H. pirai Arech. & \\
\hline Gomphrena graminea Moq. & 0,03 & HYPOXIDACEAE & \\
\hline Pfaffia tuberosa (Spreng.) Hicken & & Hypoxis decumbens $\mathrm{L}$. & 0,11 \\
\hline ANACARDIACEAE & & IRIDACEAE & \\
\hline Schinus weimmanniifolius Mart. & 0,11 & Herbertia pulchella Sweet & \\
\hline APIACEAE & & Tigridia sp. & 0,03 \\
\hline Eryngium ciliatum Cham. \& Schlecht. & 0,14 & LAMIACEAE & \\
\hline E. eriophorum Cham. \& Schlecht. & 1,28 & Glechon ciliata Benth. & \\
\hline E. horridum Malme & 3,75 & G. mariifolia Benth. & 0,11 \\
\hline E. pristis Cham \& Schlecht. & 4,67 & Glechon sp. & 0,03 \\
\hline E. sanguisorba Cham. \& Schlecht. & 1,45 & Hyptis mutabilis (L.C. Rich.) & \\
\hline APOCINACEAE & & Rhabdocaulon cf. coccineum (Benth.) Eplir & \\
\hline Mandevilla coccinea (Hook. \& Arn.) Woodson & & R. cf. stricta Benth. & \\
\hline ASCLEPIADACEAE & & LINACEAE & \\
\hline Araujia sp. & & Cliococca sellaginoides (Lam.) Rog. \& Mildr & n. 0,14 \\
\hline ASTERACEAE & & LYTHRACEAE & \\
\hline Acanthospermum australe (Loefl.) O.K. & & Cuphea sp. & \\
\hline Achyrocline satureoides (Lam.) DC. & & MALVACEAE & \\
\hline Aspilia montevidensis (Spreng.) O.K. & 0.99 & Krapovickasia macrodon (DC.) Frixell & 0,07 \\
\hline A. pascalioides Griseb. & 0.39 & Pavonia hastata Cav. & \\
\hline Baccharis articulata (Lam.) Pers. & 0,11 & Pavonia sp. 1 & \\
\hline B. caprariefolia DC. & 1,06 & Pavonia sp. 2 & \\
\hline B. crispa Spreng. & 0.42 & Sida rhombifolia $\mathrm{L}$. & \\
\hline B. dracunculifolia DC. & 0.07 & MELASTOMATACEAE & \\
\hline B. leptophylla DC. & 0,07 & Tibouchina sp. & 0,53 \\
\hline B. leucopappa DC. & 0,88 & MYRTACEAE & \\
\hline B. ochracea Spreng. & 0,25 & Myrciaria tenella Berg. & \\
\hline B. patens Baker & & Myrtaceae 1 & \\
\hline B. pseudotemuifolia Teodoro & & Myrtaceae 2 & \\
\hline B. rufescens Spreng. & 0,18 & OENOTHERACEAE & \\
\hline B. sessiliflora Vahl & 1,52 & Oenothera indecora Camb. & 0,03 \\
\hline B. spicata (Lam.) Baillon & & ORCHIDACEAE & \\
\hline B. tenuifolia DC. & 0,32 & Habenaria sp. 1 & \\
\hline B. trimera (Less.) DC. & 0,11 & Habenaria sp. 2 & \\
\hline Calea serrata Less. & 0,03 & OXALIDACEAE & \\
\hline C. uniflora Less. & 1,24 & Oxalis conorrhiza (Fuilee) Jacquin & 0,03 \\
\hline Chaptalia integerrima (Vell.) Burk. & & O. brasiliensis Loddiges & \\
\hline C. nutans (L.) Polak. & & PLANTAGINACEAE & \\
\hline C.piloselloides (Vahl) Baker & 0,18 & Plantago sp. & 0,03 \\
\hline C. simuata (Less.) Baker & 0,03 & POACEAE & \\
\hline Chevreulia acuminata Less. & 0,11 & Agenium villosum (Nees) Pilger & 8,13 \\
\hline Coniza blakei (Cabr.) Cabr. & & Agrostis montevidensis Spreng. ex Nees & \\
\hline C. cf. lorentzii Griseb. & & $\begin{array}{l}\text { Andropogon glaucophyllus Roseng., } \\
\text { Arr. \& Izag. }\end{array}$ & 0,07 \\
\hline C. chilensis Spreng. & & A. lateralis Nees & 7,35 \\
\hline Eupatorium ascendens Schultz - Bip. & & A. selloanus Hackel & 0,25 \\
\hline E. congestum Hook. \& Arn. & & Aristida circinalis Lindmann & 0,18 \\
\hline E. intermedium DC. & 1,02 & A. filifolia (Arech.) Herter & 0,32 \\
\hline E. lanigerum Hook. \& Arn. & & A. flaccida Trin. \& Rupr. & 0,03 \\
\hline
\end{tabular}




\begin{tabular}{|c|c|c|c|}
\hline Spp. & FA & Spp. & FA \\
\hline Eupatorium ligulifolium Hook. \& Arn. & 0,25 & Aristida jubata (Arech.) Hert. & 0,07 \\
\hline E. subhastatum Hooker \& Arnott & 0,03 & A. Iaevis (Nees) Kunth & 1,02 \\
\hline E. tanacetifolium Gill. ex Hook. \& Arn. & 0,64 & Aristida sp. & \\
\hline E. ulei Hieronymus & & A. spegazzinii Arech. & 1,06 \\
\hline Gamochaeta falcata (Lam.) Cabr. & 0,03 & Axonopus affinis Chase & 0,03 \\
\hline G. filaginea (DC) Cabr. & & A. argentinus Parodi & 2,4 \\
\hline Heterothalamus alienus (Spreng.) OK. & & A. cf. siccus (Nees) Kuhlm. & 1,9 \\
\hline Hieracium commersonii Monn. & 0,03 & Axonopus sp. & 0,18 \\
\hline Holocheilus brasiliensis (L.) Cabr. & 0,03 & A. suffultus (Mik. ex Trin.) Parodi & 2,26 \\
\hline Hysterionica filiformis (Spreng.) Cabr. & 0,07 & Bothriochloa laguroides (DC.) Pilger & 0,18 \\
\hline H. pinniifolia (Poir.) Baker & & Briza calotheca (Trin.) Hackel & 0,78 \\
\hline Lucilia acutifolia (Poir.) Cass. & 0,11 & B. lamarckiana Nees & 0,03 \\
\hline Mikania pinnatiloba $\mathrm{DC}$. & & B. macrostachya (Presl.) Steudel & 0,07 \\
\hline M. temuifolia DC. & 0,21 & B. poaemorpha (Presl.) Henr. & 0,21 \\
\hline \multicolumn{2}{|c|}{ Noticastrum gnaphalioides (Baker) Cuatrecasas } & B. subaristata Lam. & 1,06 \\
\hline N. marginatum (HBK) Cuatrecasas & 0,21 & B. uniolae (Nees) Steudel & 0,03 \\
\hline Orthopappus angustifolius (Suartz) Gleason & 0,03 & Bromus auleticus Trin. ex Nees & 0,07 \\
\hline Porophyllum obscurum (Spreng.) DC. & & Calamagrostis alba (Presl) Steudel ssp. alba & 0,11 \\
\hline P. ruderale (Jacq.) Cass. & 0,39 & C. viridiflavescens (Poir.) Steud. & \\
\hline Pterocaulon alopecuroides (Lam.) DC. & 0,14 & Chloris distichophylla Lag. & \\
\hline P. angustifolium $\mathrm{DC}$. & & C. retusa Lag. & \\
\hline P. bakeri Malme & 0,15 & C. retusa Lag. & \\
\hline P. internuptum DC. & 0,14 & C. uliginosa Hack. & 0,03 \\
\hline P. lorentzii Malme & 0,28 & Danthonia cirrata Hack. \& Hack. & 0,14 \\
\hline P. rugosum (Vahl) Malme & & D. montevidensis Hack. \& Arech. & \\
\hline Schlechtendalia luzulaefolia Less. & 0,21 & Danthonia sp. & 0,03 \\
\hline Senecio brasiliensis (Spreng.) Less. & & Elyonurus candidus (Trin.) Hack. & 3,36 \\
\hline S. cisplatinus Cabr. & 0,11 & E. rostratus Nees & 0,60 \\
\hline S. heterotrichus DC. & 0,03 & Eragrostis lugens Nees & 0,11 \\
\hline S. selloi (Spreng.) DC. & 0,78 & E. neesii Trin. var. neesii & 0,03 \\
\hline Senecio sp. & & E. neesii var. lindmanii (Hack.) Ekman & \\
\hline Solidago chilensis Meyen & 0,07 & E. polytricha Nees & 0,14 \\
\hline Spilanthes grisea (Chod.) A. H. Moore & & $\begin{array}{l}\text { Gymnopogon burchelii (Munro ex Doell) } \\
\text { Ekman }\end{array}$ & 0,11 \\
\hline Stenachaenium riedelli Baker & 0,03 & Leptochoryphium lanatum (H.B.K.) Nees & 0,03 \\
\hline Stevia cinerascens Shultz - Bip. ex Baker & & Melica brasiliana Arduinus & 0,14 \\
\hline Symphyopappus reticulatus Baker & 0,21 & M. eremophila M.A. Torres & \\
\hline Tagetes minuta $\mathrm{L}$. & & Melinis minutiflora Beauv. & \\
\hline Trixis stricta (Spreng.) Less. & 0,25 & Microchloa indica (L.f.) Beauv. & 0,07 \\
\hline T. verbaciformis Less. & & Panicum bergii Arech. & 0,21 \\
\hline Verbesina subcordata DC. & & P. olyroides H.B.K. & 0,39 \\
\hline Vernonia brevifolia Less. & 0,07 & P. peladoense Henr. & 0,21 \\
\hline V. cognata Less. & & P. sabulorum Lam. & 0,18 \\
\hline V.flexuosa Sims & 0,28 & Panicum sp. & 0,18 \\
\hline V. megapotamica Spreng. & 0,14 & Paspalum ionanthum Chase & 0,07 \\
\hline V. nudiflora Less. & 0,88 & P. notatum $\mathrm{Fl}$. & 0,03 \\
\hline V. rubricaulis Humb. \& Bonpl. & 0,03 & P. plicatulum Michx. & 1,80 \\
\hline V. sellowii Less. & & P. polyphyllum Nees ex Trin. & 0,03 \\
\hline BORAGINACEAE & & Paspalum sp. & 0,03 \\
\hline Moritzia ciliata (Cham.) DC. & 0,21 & Piptochaetium lasianthum Griseb. & 0,32 \\
\hline BROMELIACEAE & & P. montevidense (Spreng.) Parodi & 0,39 \\
\hline Dichya sp. & & P. ruprechtianum Desv. & 0,03 \\
\hline CACTACEAE & & P. stipoides (Trin. et Ruprecht.) Hack. & \\
\hline
\end{tabular}




\begin{tabular}{|c|c|c|c|}
\hline Spp. & FA & Spp. & FA \\
\hline Notocactus ottonis A. Berger & 0,03 & Poa lanigera Nees & 0,03 \\
\hline CAMPANULACEAE & & Polypogon chilensis (Kunth) & 0,03 \\
\hline Whalenbergia linarioides (Lam.) DC. & 0,03 & Rhynchelytrum repens (Willd.) Hubb. & 0,49 \\
\hline CARYOPHYLLACEAE & & Schizachyrium gracilipes (Hack.) Camus & 0,49 \\
\hline Cardionema ramosissima (Weinm.) Nels \& M & acb. & S. imberbe (Hack.) Camus & 2,51 \\
\hline Paronychia chilensis DC. & & $\begin{array}{l}\text { S. microstachyum (Desv.) Roseng. ssp. elong } \\
\text { (Hack.) Roseng. }\end{array}$ & gatum \\
\hline CISTACEAE & & $\begin{array}{l}\text { S. microstachyum (Desv.) Roseng. ssp. } \\
\text { microstachyum }\end{array}$ & 0,18 \\
\hline Helianthemum brasiliense (Lam.) Persoon & & Schizachyrium sp. & \\
\hline COMMELINACEAE & & $\begin{array}{l}\text { S. spicatum (Spreng.) Herter ssp. } \\
\text { breviarticulatum }\end{array}$ & 0,25 \\
\hline Commelina sp. & & S. tenerum Nees & 8,27 \\
\hline CONVOLVULACEAE & & Setaria geniculata (Lam.) Beauv. & 0,25 \\
\hline Evolvulus sp. & 0,03 & S. vaginata Spreng. & \\
\hline CYPERACEAE & & Sorghastrum albescens (Hack.) Flores & 3,32 \\
\hline $\begin{array}{l}\text { Bulbostylis capillaris (L.) C.B.Clarke } \\
\text { var. elatior (Griseb.) Osten }\end{array}$ & 0,28 & Sporobolus camporum Swallen & 0,03 \\
\hline B. cf. closii Barros & & $S$. indicus (L.) R.Br. & 0,03 \\
\hline B. consangiuinea (Kunth) C.B.Clarke & 0,07 & Stipa filiculmis Delile & 0,07 \\
\hline B. cf. juncoides (Vahl) Kük. & 0,07 & S. filifolia Nees & 0,67 \\
\hline B. sphaerocephala (Boeck.) C.B. Clarke & 0,03 & S. megapotamia Spreng. ex Trin. & \\
\hline Cyperus cayennensis (Lam.) Britton & & S. mela & \\
\hline C. reflexus Vahl & & is Hack. & 0,07 \\
\hline Fimbristylis juncoides (Vahl) Alain & & Stipe & 0,03 \\
\hline Rhynchospora barrosiana Guaglianone & 0,11 & S. tenuiculmis Hack. & 0,18 \\
\hline R. globosa Roem. et Schult. & & $\begin{array}{l}\text { Trachypogon montufari (H.B.K.) } \\
\text { Nees var. montufari }\end{array}$ & 8,38 \\
\hline R. luzuliformis Boeck. & 0,18 & $\begin{array}{l}\text { T. montufari (H.B.K.) Nees var. mollis } \\
\text { (Nees) Burk. }\end{array}$ & 0,03 \\
\hline R. setigera (Kunth) Boeck. & 0,57 & POLYGONACEAE & \\
\hline EUPHORBIACEAE & & Monnina oblongifolia Arech. & \\
\hline Croton gnaphalli Baill. & 0,14 & Polygalas & 0,03 \\
\hline Croton sp. & & ACEAE & \\
\hline Euphorbia selloi (KI. \& Garcke) Boissier & 0,25 & Anagallis arvensis $\mathrm{L}$. & \\
\hline Tragia sp. & & RHAMNACEAE & \\
\hline FABACEAE & & Colletia paradoxa (Spreng.) Escal. & \\
\hline Aeschynomene falcata (Poir.) DC. & 0,15 & RUBIACEAE & \\
\hline Centrosema virginianum (L.) Benth. & 0,03 & $\begin{array}{l}\text { Borreria capitata (Ruiz \& Pav.) DC. var, ten } \\
\quad \text { (H.B.K.) Steyerm. }\end{array}$ & nella \\
\hline Chamaecrista flexuosa (L.) Greene & & B. capitata (Ruiz \& Pav.) DC. & 0,07 \\
\hline $\begin{array}{l}\text { C. nictitans (L.) Moench. subsp. patellaria } \\
\text { (DC. ex. Col.) Irwin \& Barneby }\end{array}$ & & B. fastigiata (Gris.) Schum. & 0,28 \\
\hline C. repens (Vogel) Irwin \& Barneby & 0,42 & oides Cham. \& Schlecht. & 0,14 \\
\hline Clitoria nana Benth. & 0,07 & B. verticillata (L.) G.F.W. Meyer & 0,03 \\
\hline Collaea stenophylla (Hook. \& Arn.) Benth. & 0,60 & Diodia apiculata (R. \& S.) Schum. & \\
\hline Crotalaria tweediana Benth. & 0,39 & $\begin{array}{l}\text { Richardia grandiflora (Cham. \& Schlecht.) } \\
\text { Steud. }\end{array}$ & 0,49 \\
\hline Desmanthus v & 0,18 & R. humistrata (Cham. \& Schlecht.) Steud. & 0,07 \\
\hline D. incanum (Sw.) DC. & 0,57 & R. stellaris (Cham. \& Schlecht.) Steud. & 0,03 \\
\hline D. uncinatum (Jacq.) DC. & & SCROPHULARIACEAE & \\
\hline Eriosema tacuaremboense Arech. & 0,32 & Angelonia integerrima Spreng. & \\
\hline Galactia marginalis Benth. & 0,07 & Gerardia communis Cham. \& Schlecht. & \\
\hline
\end{tabular}




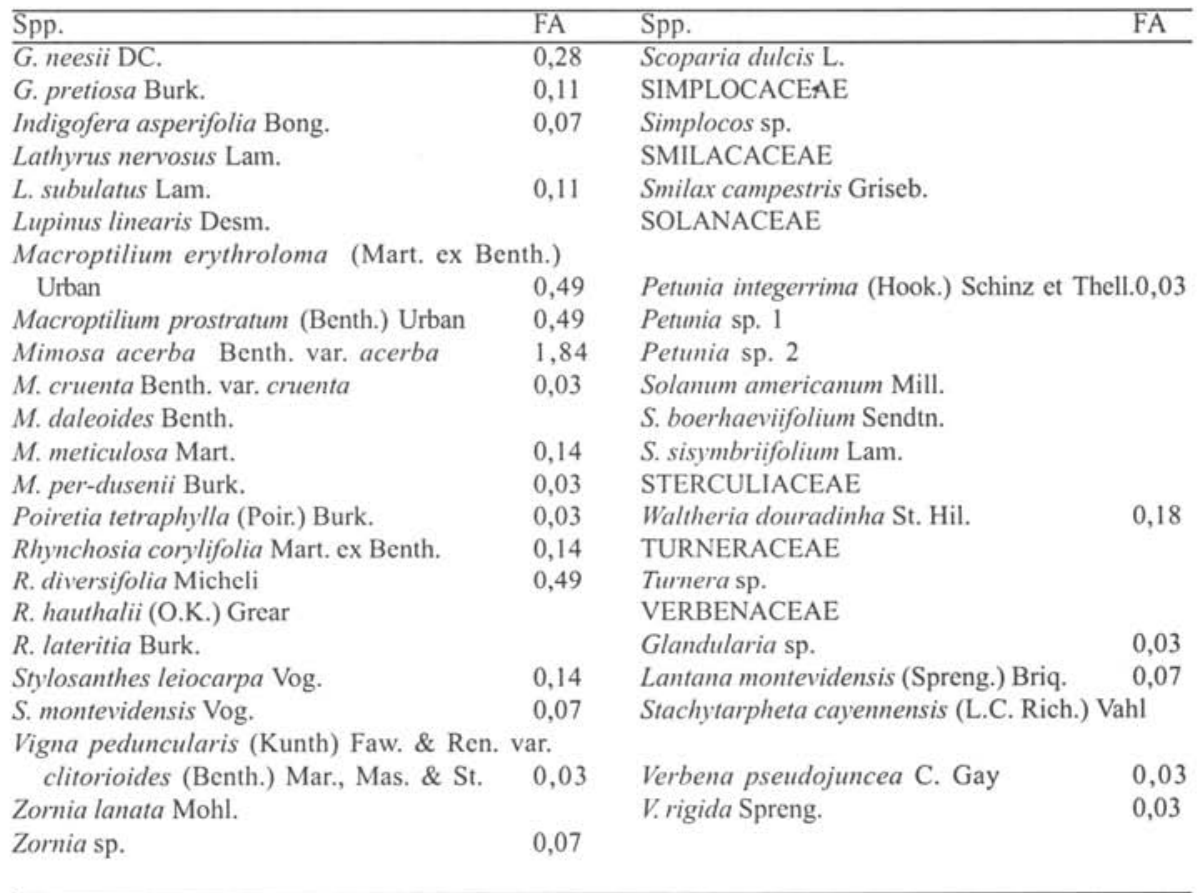

Os valores de riqueza e diversidade encontrados neste trabalho são superiores aos obtidos por outros autores em vegetação campestre no Rio Grande do Sul. Por exemplo, Landau (1994), estudando vegetação campestre antropogênica, encontrou valores de diversidade $(\mathrm{H})$ variando de 1,01 a 2,26. Boldrini (1993) registrou um total de 256 espécies $(R=5,55)$ numa área de 30 ha de vegetação campestre natural. Bueno et al. (1979) encontraram diversidade $(\mathrm{H})$ de 2,01 em campo seco, 1,34 em campo úmido e 1,71 em campo encharcado.

Composição floristica e exposição solar - as cinco espécies com maior freqüência absoluta nas diferentes exposições estão relacionadas na Tab. 2 e mostram claramente o predominio das gramíneas.

Tipos de vegetação e fatores de ambiente - a análise de agrupamentos foi a que revelou grupos mais coerentes com a variação dos fatores de ambiente. No dendrograma observam-se dois grupos bem nitidos: o primeiro que reúne as comunidades dispostas no lado sul e oeste e o segundo formado pelas comunidades da porção norte e leste (Fig. 2).

A análise de ordenação revelou configuração das comunidades mais coerente com a variação dos fatores de ambiente. No diagrama de dispersão de comunidades (Fig. 3), os componentes (eixos) I e II explicam $42 \%$ da variação total da vegetação. Verifica-se no diagrama que as comunidades localizadas à direita são aquelas com 
Tabela 2. Frequiência absoluta (FA) das espécies dominantes na vegetação campestre do Morro da Polícia, nas diferentes exposições.

\begin{tabular}{lrl}
\hline EXPOSIÇAOO & FA(\%) & ESPECIES \\
\hline NORTE & 13.70 & Trachypogon montufari \\
& 7.91 & Schizachyrium tenerum \\
& 6.92 & Andropogon lateralis \\
& 4.66 & Agenium villosum \\
& 4.52 & Axonopus suffultus \\
LESTE & 8.66 & Schizachyrium tenerum \\
& 7.77 & Trachypogon montufari \\
& 6.33 & Eryngium pristis \\
& 4.88 & Sorghastrum albescens \\
& 3.77 & Elyonurus candidus \\
& 16.94 & Agenium villosum \\
& 9.65 & Schizachyrium tenerum \\
& 9.23 & Trachypogon montufari \\
& 5.81 & Axonopus argentinus \\
& 4.71 & Andropogon glaucophyllus / A. lateralis \\
& 13.30 & Agenium villosum \\
& 7.40 & Schizachyrium tenerum \\
& 6.77 & Andropogon lateralis \\
& 6.52 & Trachypogon montufari \\
& 4.58 & Rhynchospora setigera \\
\hline
\end{tabular}

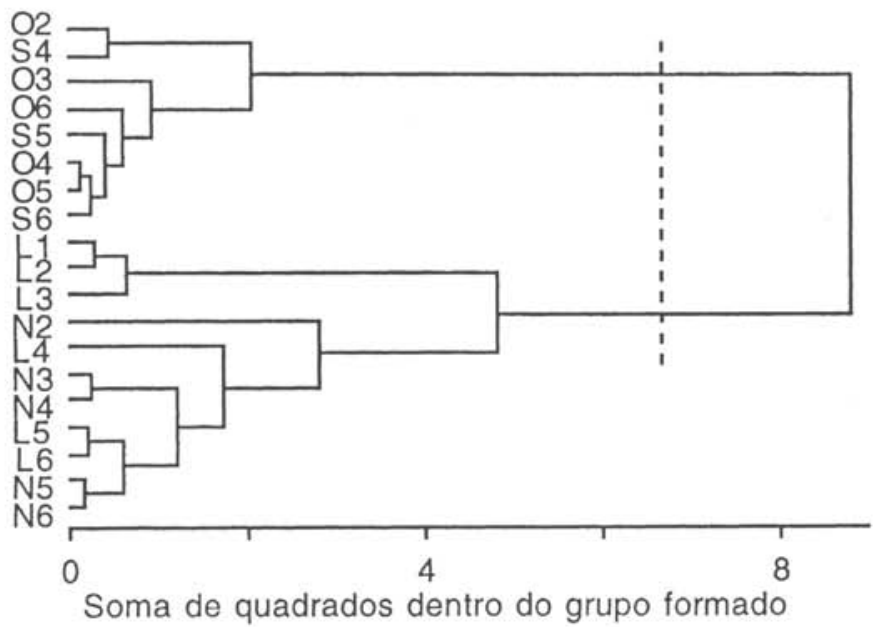

Figura 2. Dendrograma obtido por análise de agrupamentos de unidades amostrais, vegetação herbácea, Morro da Polícia, Porto Alegre, pelo critério de variância mínima (soma de quadrados), a partir de uma matriz de distâncias de corda. As unidades amostrais estão identificadas pela exposição solar (N, S, L, O) e classe de altitude (1-6). 
exposição norte e leste, portanto mais expostas à radiação solar, enquanto que as localizadas à esquerda são as comunidades que estão na exposição oeste e sul, submetidas a menor radiação solar, o que nos leva a concluir que o eixo horizontal (componente I) está relacionado com exposição solar. A explicação da variação da vegetação através deste eixo é de $25,9 \%$, o que pode ser considerado um valor alto, esperado em vista da complexidade da composição florística. Essa configuração de comunidades no diagrama corrobora o resultado obtido na análise de agrupamentos (Fig. 3).

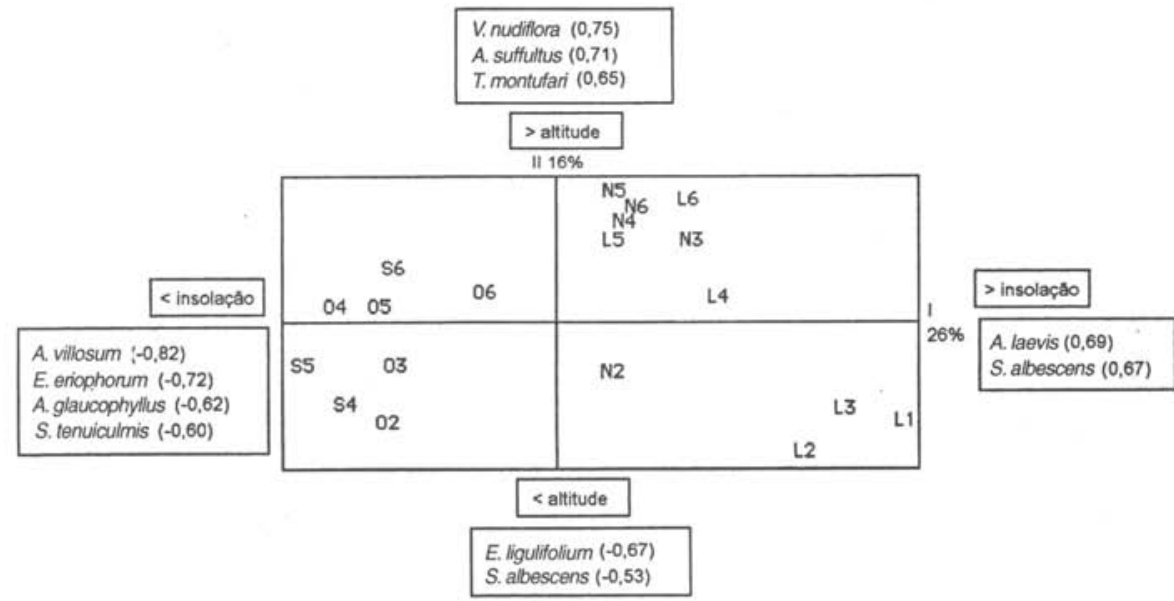

Figura 3. Diagrama de dispersão de unidades amostrais, vegetação herbácea, Morro da Polícia, Porto Alegre, gerado por análise de coordenadas principais a partir de uma matriz de distâncias de corda com os dados de freqüência absoluta das espécies. As unidades amostrais estão identificadas pela exposição solar $(\mathrm{N}, \mathrm{S}, \mathrm{L}, \mathrm{O}) \mathrm{e}$ classe de altitude (1-6). Espécies que apresentaram coeficientes de correlação mais altos com os eixos da ordenação estão indicadas no diagrama, sendo as correlações ajustadas à escala do diagrama. Por exemplo, Agenium villosum (Agvi) apresenta correlação negativa e alta $(r=-0,83)$ com o eixo I e positiva e baixa $(r=0,14)$ com o eixo II. As demais espécies indicadas são Andropogon lateralis (Anla), Vernonia nudiflora (Venu), Tachypogon muntufari (Trmo), Axonopus suffultus (Axsu), Aristida laevis (Arla), Sorghastrum albescens (Soal), Eryngium eriophorum (Erer), Stipa tenuiculmis (Stte) e Eupatorium ligulifolium (Euli). As tendências de variação em relação aos fatores altitude e insolação foram reveladas a partir da interpretação do diagrama.

As espécies que apresentam altos coeficientes de correlação positivos com o eixo I da ordenação (aumentam da esquerda para a direita no diagrama da Fig. 3) são: Aristida laevis $(\mathrm{r}=0,69)$ e Sorghastrum albescens $(\mathrm{r}=0,72)$, as quais portanto caracterizam as comunidades mais expostas à radiação solar. Também correlacionadas positivamente com o eixo I, destacam-se Baccharis leptophylla $(\mathrm{r}=0,48)$, Stipa filifolia $(\mathrm{r}=0,46)$ e Axonopus siccus $(\mathrm{r}=0,40)$. As que apresentam altos coeficientes de correlação negativos, ou seja, as espécies associadas com menor intensidade ou menor quantidade de insolação, são: Agenium villosum $(\mathrm{r}=-0,83)$, Eryngium eriophorum 
( $\mathrm{r}=-0,76)$, Andropogon glaucophyllus $(\mathrm{r}=-0,67)$ e Stipa tenuiculmis $(\mathrm{r}=-0,60)$. Outras espécies, também correlacionadas negativamente com o eixo I, são Axonopus argentinus $(\mathrm{r}=-0,55)$, Schizachyrium imberbe $(\mathrm{r}=-0,53)$ e Calamagrostis alba $(\mathrm{r}=-0,52)$.

Quanto à associação entre vegetação e altitude, o diagrama de dispersão (Fig. 3) mostra que as comunidades localizadas na parte superior do mesmo, correspondem às situadas em posições mais elevadas do morro. Na porção inferior do diagrama estão distribuídas as que se situam nas partes mais baixas, com exceção das unidades amostrais S4 e S5. Neste caso, provavelmente, a altitude elevada, onde estão localizadas estas comunidades, está sendo compensada pela menor insolação, já que a exposição é sul.

O eixo vertical (componente II) explica $16 \%$ da variação da vegetação e está relacionado, como já referido, com a altitude. As espécies com altos coeficientes de correlação positivos com o eixo II correspondem a Vernonia nudiflora $(r=0,74)$, Axonopus suffultus $(\mathrm{r}=0,73)$ e Trachypogon montufari $(\mathrm{r}=0,73)$. Essas são as espécies que caracterizam as maiores altitudes. Além dessas, ocorrem outras espécies com coeficientes mais baixos: Andropogon lateralis $(\mathrm{r}=0,66)$, Aspilia montevidense $(0,59)$, Andropogon selloanus $(\mathrm{r}=0,59)$, Panicum sp. $(\mathrm{r}=0,57)$, Setaria geniculata $(\mathrm{r}=0,54)$, Calea uniflora $(\mathrm{r}=0,56)$, Croton gnaphalii $(0,53)$ e Vernonia flexuosa $(\mathrm{r}=0,52)$ e Eryngium sanguisorba $(\mathrm{r}=0,49)$.

Os maiores coeficientes de correlação negativos com o eixo II correspondem a Eupatorium ligulifolium $(\mathrm{r}=-0,66)$ e Sorghastrum albescens $(\mathrm{r}=-0,49)$, as quais caracterizam as menores altitudes. Além dessas, com correlações mais baixas estão Andropogon glaucophyllus $(\mathrm{r}=-0,42)$, Eryngium eriophorum $(\mathrm{r}=-0,37)$ e Rhynchospora setigera $(\mathrm{r}-0,34)$.

\section{Referências bibliográficas}

Aguiar, L.W.; Martau, L.; Soares, Z.F.; Bueno, O.L.; Mariath, J.E. \& Klein, R.M. 1986. Estudo preliminar da flora e vegetação de morros graníticos da região da grande Porto Alegre, Rio Grande do Sul, Brasil. Iheringia 34: 3-38.

Boldrini, I. I. 1993. Dinâmica de vegetação de uma pastagem natural submetida a níveis de oferta de forragem e tipos de solo, Depressão Central, RS. Universidade Federal do Rio Grande do Sul, Porto Alegre. Tese de Doutorado.

Borges Fortes, A. 1959. Geografia física do Rio Grande do Sul. Gráfica da Livraria do Globo, Porto Alegre.

Bueno, O. L.; Buselato, T. C. \& Miotto, S. T. S. 1979. Composição florística de um campo localizado no município de Montenegro, RS, Brasil. In: Anais do XXX Congresso Nacional de Botânica, Campo Grande 1979. São Paulo, Sociedade Botânica do Brasil V. 1, p. 5-21.

Landau, E. C. 1994. Ecologia de paisagem da região do Vale do Rio dos Sinos - Santa Cristina do Pinhal, Parobé, RS, em bases fitossociológicas e de geoprocessamento. Universidade Federal do Rio Grande do Sul, Porto Alegre. Dissertação de Mestrado.

Levy, E. B. \& Madden, E.A. 1933. The point method of pasture analysis. New Zealand Journal of Agriculture 46: $267-279$.

Mantovani, W. \& Martins, F.R. 1990. O método de pontos. Acta Botanica Brasilica 4 (2): 95-122.

Mohr, F.V. 1995. Zoneamento da vegetação da Reserva Ecológica do Morro Santana - Porto Alegre, RS. Universidade Federal do Rio Grande do Sul, Porto Alegre. Dissertação de Mestrado.

Pielou, E. C. 1975. Ecological Diversity. Wiley, New York.

Pielou, E. C. 1984. The Interpretation of Ecological Data. Wiley, New York.

Pillar, V. D. P. 1995. SYNCSA: software para análise multivariada baseada em caracteres e testes de aleatorização. Departamento de Botânica, Universidade Federal do Rio Grande do Sul, Porto Alegre. 
Pillar, V. D. P. \& Orlóci, L. 1993. Character-Based Community Analysis: The Theory and an Application Program. SPB Academic Publishers, Haia, Holand.

Podani, J. 1994. Multivariate Data Analysis in Ecology and Systematics. SPB Academic Publishers, Haia, Holand.

Rambo, B. 1954. Análise histórica da flora de Porto Alegre. Sellowia 6: 9-111.

Rambo, B. 1956. A fisionomia do Rio Grande do Sul, $2^{a}$ ed., Selbach, Porto Alegre.

Teixeira, M.B.; Coura-Neto, A.B.; Pastore, V. \& Rangel-Filho, A.L.R. 1986. Vegetação: as regiões fitoecológicas, sua natureza e seus recursos econômicos, estudo fitogeográfico. In: Folha SH.22 Porto Alegre e parte das folhas SH.21 Uruguaiana e SI.22 Lagoa Mirim. Rio de Janeiro: IBGE (Levantamento de Recursos Naturais, v. 33).

Teodoro L. 1960. Flora analítica de Porto Alegre . Instituto Geobiológico La Salle, Canoas. s.p. 